\title{
ASYMPTOTIC PRIME DIVISORS AND ANALYTIC SPREADS
}

\author{
STEPHEN MCADAM ${ }^{1}$
}

\begin{abstract}
Let $I$ be an ideal in a Noetherian domain $R$, and let $\hat{I}$ be the integral closure of $I$. Let $\hat{A}^{*}(I)=\operatorname{Ass}\left(R / \hat{I}^{n}\right)$ for $n$ large (it being known that for large $n$ this set does not vary with $n$ ). Suppose that $R$ satisfies the altitude formula. Then it is shown that $P \in \hat{A}^{*}(I)$ if and only if height $P=l\left(I_{P}\right)$, the analytic spread of $I_{P}$.
\end{abstract}

Introduction. Let $I$ be an ideal in a Noetherian ring. For $n>1$, let $A(n)$ be the set of prime divisors of $I^{n}, A(n)=\operatorname{Ass}\left(R / I^{n}\right)$. A recent paper of Brodmann [1] shows that $A(n)$ is constant for $n$ large. In [5] that constant is denoted $A^{*}=A^{*}(I)$. In general it is difficult to explicitly determine $A^{*}$ for a given ideal $I$, although in [5, Corollary 22] this is done for $R$ a 2-dimensional normal domain. This paper will discuss a concept related to $A^{*}$, namely $\hat{A}^{*}$. Let $\hat{I}$ denote the integral closure of the ideal $I$, and let $\hat{A}(n)=\operatorname{Ass}\left(R / \hat{I}^{n}\right)$, the prime divisors of $\hat{I}^{n}$. If height $I>1,[5$, Proposition 7] shows that $\hat{A}(n)$ is constant for large $n$. That constant is denoted by $\hat{A}^{*}=\hat{A}^{*}(I)$. The purpose of this paper is to characterize $\hat{A}^{*}$ for any ideal $I$ in a Noetherian domain satisfying the altitude formula. The characterization is $P \in \hat{A}^{*}$ if and only if height $P=l\left(I R_{P}\right)$, the analytic spread of $I R_{P}$.

Preliminaries. Throughout this paper, $R$ will denote a Noetherian domain, $I$ an ideal of $R$, and $P$ a prime ideal of $R$ containing $I$. The domain $T$ will always be $T=R[I x]=R+I x+I^{2} x^{2}+\ldots, x$ an indeterminate. Since $T \subset R[x]$, obviously the transcendence degree of $T$ over $R$ is 1 . We will occasionally mention the form ring of $I, R / I+I / I^{2}+\ldots$ Note that this is isomorphic to $T / I T$. If $(R, P)$ is local, we will also use the ring $R / P+I / P I+I^{2} / P I^{2}+\ldots$, which is isomorphic to $T / P T$. Finally, $P^{\prime \prime}$ will be $P+I x+I^{2} x^{2}+\ldots$ in $T$.

If $(R, P)$ is a local domain and $I$ is an ideal of $R$, then $l(I)$ denotes the analytic spread of $I$. Recall that there are various characterizations of $l(I)$. (i) If $R / P$ is infinite and if $J$ is a minimal reduction of $I$ then $l(I)=v(J)$, the minimal number of generators of $J$. (ii) $l(I)=\operatorname{height}\left(P^{\prime \prime} / P T\right)$. (See [7] and [8] for basics on reductions and $l(I)$.) Also by the altitude inequality (stated below) height $P+$ $\operatorname{TRD}(T / R)>$ height $P^{\prime \prime}+\operatorname{TRD}\left(P^{\prime \prime} / P\right)$ giving height $P+1>$ height $P^{\prime \prime}>$ height $\left(P^{\prime \prime} / P T\right)=l(I)$. Thus height $P>l(I)$. (See [2] for more.)

Received by the editors July 10, 1979.

AMS (MOS) subject classifications (1970). Primary 13E05; Secondary 13A15.

Key words and phrases. Noetherian domain, prime divisors, analytic spread, integral closure of an ideal.

${ }^{1}$ Partially supported by the National Science Foundation. 
Let the domain $S$ be a finitely generated ring extension of $R$. Let $Q$ be prime in $S$ with $Q \cap R=P$. It is well known that height $P+\operatorname{TRD}(S / R)>$ height $Q+$ $\operatorname{TRD}(Q / P)$. Here "TRD" denotes transcendence degree and $\operatorname{TRD}(Q / P)$ refers to the transcendence degree of $S / Q$ over $R / P$. If in fact height $P+\operatorname{TRD}(S / R)=$ height $Q+\operatorname{TRD}(Q / P)$ for all such $S$ and $Q$, then $R$ is said to satisfy the altitude formula. Almost all known Noetherian domains do satisfy the altitude formula. The only known counterexamples are variations on [6, Example 2, pp. 202-205]. Thus assuming that the altitude formula holds is a minor restriction.

$\hat{A}^{*}$ and $l(I)$. Our first lemma is essentially a restatement of [5, Proposition 18] in a more efficient manner.

LEMMA 1. Let $R$ be a Noetherian domain which satisfies the altitude formula. Let $0 \neq I \subseteq P$ be ideals of $R$, with $P$ prime. Then $P \in \hat{A}^{*}$ if and only if there is a height one prime $P^{\prime}$ of $T=R+I x+I^{2} x^{2}+\ldots$, with $P^{\prime} \cap R=P$. If this is the case, then $P^{\prime}$ is homogeneous.

Proof. Suppose first that such a $P^{\prime}$ exists. As $I \subseteq P \subset P^{\prime}, I T \subseteq P^{\prime}$ and so in the form ring of $I, T / I T, P^{\prime} / I T$ is a minimal prime. According to [5, Proposition 18], in order to show that $P \in \hat{A}^{*}$ we need only show that $P^{\prime} / I T$ is a relevant prime. Being a minimal prime, $P^{\prime} / I T$ is homogeneous (thus $P^{\prime}$ is homogeneous as stated), and so if it is not relevant then clearly $P^{\prime}=P+I x+I^{2} x^{2}+\ldots$, so that $T / P^{\prime}=R / P$. Applying the altitude formula to $R \subset T$ and the primes $P$ and $P^{\prime}$ gives height $P+\operatorname{TRD}(T / R)=$ height $P^{\prime}+\operatorname{TRD}\left(P^{\prime} / P\right)$, that is, height $P+1=$ $1+0$. Thus height $P=0$ contradicting that $0 \neq I \subseteq P$. Therefore $P^{\prime} / I T$ is relevant, as required.

Conversely, suppose that $P \in \hat{A}^{*}$. By [5, Proposition 18], in the form ring $T / I T$ there is a minimal prime, call it $P^{\prime} / I T$, with $\left(P^{\prime} / I T\right) \cap(R / I)=P / I$. To prove the lemma, we must only show that in $T$, height $P^{\prime}=1$. We go to the Rees ring $T+x^{-1} R\left[x^{-1}\right]$, and consider the prime $P^{\prime}+x^{-1} R\left[x^{-1}\right]$. Since $T$, being a finitely generated extension of $R$, satisfies the altitude formula, and since $T / P^{\prime}=$ $\left(T+x^{-1} R\left[x^{-1}\right]\right) /\left(P^{\prime}+x^{-1} R\left[x^{-1}\right]\right)$ we have height $P^{\prime}=$ height $P^{\prime}+$ $x^{-1} R\left[x^{-1}\right]$. As $P^{\prime}$ is minimal over $I T, P^{\prime}+x^{-1} R\left[x^{-1}\right]$ is minimal over $I T+$ $x^{-1} R\left[x^{-1}\right]=x^{-1}\left(T+x^{-1} R\left[x^{-1}\right]\right)$, which is a principal ideal of the Rees ring. Accordingly, height $P^{\prime}=1$.

COROllary 2. Let $(R, P)$ be a local domain satisfying the altitude formula. Let $I$ be an ideal of $R$. Then $P \in \hat{A}^{*}$ if and only if $P T$ is a height one ideal of $T$.

Proof. If $P \in \hat{A}^{*}$, pick $P^{\prime}$ as in the lemma. Obviously $P T \subseteq P^{\prime}$ and so height $P T=1$. Conversely if height $P T=1$, let $P^{\prime}$ be a height one prime of $T$ containing $P T$. Thus $P \subset P T \subseteq P^{\prime}$ and so $P^{\prime} \cap R=P$. By the lemma, $P \in \hat{A}^{*}$.

THEOREM 3. Let $R$ be a Noetherian domain satisfying the altitude formula. Let $I \neq 0$ be an ideal of $R$ and let $P$ be a prime containing $I$. Then $P \in \hat{A}^{*}$ if and only if $l\left(I R_{P}\right)=$ height $P$. 
Proof. We may assume that $R$ is local at $P$, and write $l(I)$ for $l\left(I R_{P}\right)$. Suppose first that height $P=l(I)$, call this $n$. Let $P^{\prime \prime}$ be the prime $P+I x+I^{2} x^{2}+\ldots$ of $T=R+I x+I^{2} x^{2}+\ldots$ Since $n=l(I)=$ height $\left(P^{\prime \prime} / P T\right)$ in the ring $T / P T$, we have a chain of primes $P_{0}^{\prime} \subset P_{1}^{\prime} \subset \cdots \subset P_{n}^{\prime}=P^{\prime \prime}$ in $T$ with $P T \subseteq P_{0}^{\prime}$. Obviously $P_{0}^{\prime} \cap R=P$. In order to show that $P \in \hat{A}^{*}$, in view of the lemma we must only show that height $P_{0}^{\prime}=1$. We apply the altitude formula to $R \subset T$ and the primes $P$ and $P^{\prime \prime}$. Since height $P=n, \operatorname{TRD}(T / R)=1$, and $T / P^{\prime \prime}=R / P$, the altitude formula yields $n+1=$ height $P^{\prime \prime}$. Now the chain $P_{0}^{\prime} \subset P_{1}^{\prime} \subset \cdots \subset P_{n}^{\prime}=$ $P^{\prime \prime}$ shows that height $P_{0}^{\prime}=1$ as required.

Conversely, suppose that $P \in \hat{A}^{*}$, and now let $n=l(I)$. As above we have $P_{0}^{\prime} \subset P_{1}^{\prime} \subset \cdots \subset P_{n}^{\prime}=P^{\prime \prime}$ with $P T \subseteq P_{0}^{\prime}$. Since $P \in \hat{A}^{*}$, by Lemma 1 there is a height 1 homogeneous prime $P^{\prime}$ of $T$ with $P^{\prime} \cap R=P$. As $(R, P)$ is local, and $P^{\prime}$ is homogeneous, $P^{\prime} \subseteq P+I x+I^{2} x^{2}+\cdots=P^{\prime \prime}$. Let $k=\operatorname{height}\left(P^{\prime \prime} / P^{\prime}\right)$. As $P \subset P^{\prime}, P T \subseteq P^{\prime}$. Thus $k=\operatorname{height}\left(P^{\prime \prime} / P^{\prime}\right)<\operatorname{height}\left(P^{\prime \prime} / P T\right)=l(I)=n$. That is $k<n$. As $R$ satisfies the altitude formula, $T$ is catenary [4, Corollary 2.5] and so height $P^{\prime \prime}=$ height $\left(P^{\prime \prime} / P^{\prime}\right)+$ height $P^{\prime}=k+1$. Also height $P^{\prime \prime}=$ height $\left(P^{\prime \prime} / P_{0}^{\prime}\right)+$ height $P_{0}^{\prime}>n+1$ (by the existence of the chain $P_{0}^{\prime} \subset \cdots \subset P_{n}^{\prime}$ $=P^{\prime \prime}$ and the fact that $\left.0 \neq P T \subset P_{0}^{\prime}\right)$. Thus we have $k+1=$ height $P^{\prime \prime}>n+1$. As we previously saw $k<n$ we get $n=k$ and height $P^{\prime \prime}=n+1$. Finally the altitude formula applied to $R \subset T$ and $P$ and $P^{\prime \prime}$ gives height $P+1=$ height $P^{\prime \prime}+0$ so that height $P=n=l(I)$.

COROllary 4. Let $R$ be a Noetherian domain satisfying the altitude formula. Let $0 \neq I \subseteq P$ be ideals of $R$ with $P$ prime. If $P \in \hat{A}(n)$ for any $n>1$, then height $P=l\left(I R_{P}\right)$.

Proof. By [9, Theorem 2.5], $\hat{A}(n) \subseteq \hat{A}^{*}$, and so the corollary is immediate from Theorem 3.

We can strengthen Corollary 2 in the case that $I$ is basic. Recall that an ideal in a local domain is basic if $v(I)=l(I)$, or equivalently if $I$ is a minimal reduction of itself. (Notice that in discussing $\hat{A}^{*}$, one may always assume that $I$ is basic, since if $J$ is a minimal reduction of $I$, making $J$ basic, then for all $n>1, J^{n}$ reduces $I^{n}$ so that $\hat{J}^{n}=\hat{I}^{n}$.)

Corollary 5. Let I be a basic ideal in a local domain $(R, P)$ which satisfies the altitude formula. Then $P \in \hat{A}^{*}$ if and only if $P T$ is a height 1 prime of $T$.

Proof. Assume that $P \in \hat{A}^{*}$. We refer to the second half of the proof of Theorem 3. We have height $\left(P^{\prime \prime} / P^{\prime}\right)=k=n=l(I)=v(I)$. If $I=\left(a_{1}, \ldots, a_{n}\right)$ then $T=R\left[a_{1} x, \ldots, a_{n} x\right]$ and we have an obvious homomorphism from $R\left[x_{1}, \ldots, x_{n}\right]$ onto $T$. Let $Q^{\prime \prime}$ and $Q^{\prime}$ be the inverse images of $P^{\prime \prime}$ and $P^{\prime}$ respectively. Since $P^{\prime \prime} \cap R=P=P^{\prime} \cap R$, we have $Q^{\prime \prime} \cap R=P=Q^{\prime} \cap R$. Thus $Q^{\prime \prime}$ and $Q^{\prime}$ are two primes in $R\left[x_{1}, \ldots, x_{n}\right]$ both lying over $P$. However height $\left(Q^{\prime \prime} / Q^{\prime}\right)=\operatorname{height}\left(P^{\prime \prime} / P^{\prime}\right)=n$. This forces $Q^{\prime}$ to be $P R\left[x_{1}, \ldots, x_{n}\right]$ and so its image $P^{\prime}$ is $P T$. Thus $P T$ is a height 1 prime of $T$. The converse follows from Corollary 2. 
TheOREM 6. Let $R$ be a 2-dimensional normal Noetherian domain. Then for any ideal $I$ of $R, A^{*}=\hat{A}^{*}$.

Proof. By [9, Corollary 2.6.1], $\hat{A}^{*} \subseteq A^{*}$. Conversely, suppose that $P \in A^{*}$. If $P$ is minimal over $I$ then obviously $P \in \hat{A}^{*}$. If $P$ is not minimal over $I$ then height $P=2$. By [5, Proposition 21$], I R_{P}$ is not principal. I claim that $l\left(I R_{P}\right)>1$. If $l\left(I R_{P}\right)=1$ then by the usual method, we may assume $R_{P} / P_{P}$ is infinite, so for some $a \in R_{P}, \widehat{a R_{P}}$ reduces $I R_{P}$. Thus $a R_{P} \subseteq I R_{P} \subseteq a \hat{R}_{P}$. However, since $R_{P}$ is normal, $a R_{P}=a R_{P}$ showing that $I R_{P}$ is principal. This contradiction shows that $l\left(I R_{P}\right)>1$. We know $l\left(I R_{P}\right)<$ height $P=2$. Thus $l\left(I R_{P}\right)=2=$ height $P$. By Theorem 3, $P \in \hat{A}^{*}$, since 2-dimensional normal Noetherian domains are CohenMacaulay and hence satisfy the altitude formula [6, 35.5].

Corollary 7. Let $I$ be an ideal in a 2-dimensional normal Noetherian domain $R$. Let $P$ be prime in $R$ containing $I$. Then $P \in A^{*}$ if and only if height $P=l\left(I R_{P}\right)$.

Proof. Immediate from Theorems 3 and 6.

Corollary 7 fails without normality. If $R$ is not normal then there will always be elements $a \in R$ for which (a) $\varsubsetneqq(\hat{a})$. In our next proposition we use this to find an $I$ for which $A^{*} \neq \hat{A}^{*}$.

Proposition 8. Let $(R, P)$ be a local domain with $\operatorname{dim} R>1$. Let $0 \neq a \in R$ and suppose that $y \in(\hat{a})-(a)$. Let $I=(P y, a)$. Then for all $n>1, P \in A(n)$. If $(R, P)$ satisfies the altitude formula, then for all $n \geq 1, P \in A(n)-\hat{A}(n)$.

Proof. As $y \in(\hat{a}), y$ satisfies an equation $y^{m}+r_{1} a y^{m-1}+\cdots+r_{m} a^{m}=0$. Note $m>1$ since $y \notin(a)$. Suppose that here $m$ is the least possible. If $1<n<m$, I claim that $y^{n} \notin I^{n}$ for if $y^{n} \in I^{n}=(P y, a)^{n}$, write $y^{n}=r_{0} y^{n} p_{n}+r_{1} y^{n-1} p_{n-1} a$ $+\cdots+r_{n} p_{0} a^{n}$ with $p_{i} \in P^{i}$. Thus $y^{n}\left(1-r_{0} p_{n}\right)-r_{1} a p_{n-1} y^{n-1}-\cdots-r_{n} p_{0} a^{n}$ $=0$. This is impossible since $1-r_{0} p_{n}$ is a unit and $n<m$. Thus $y^{n} \notin I^{n}$ for $1<n<m$. Now $P^{n} y^{n} \subseteq(P y, a)^{n}=I^{n}$, and so $P^{n}$ consists of zero divisors modulo $I^{n}$. Thus $P \in A(n)$ for $1<n<m$.

I now claim that $a I^{m-1}=I^{m}$. Obviously $I^{m}=(P y)^{m}+a(P y)^{m-1}$ $+\cdots+a^{m-1} P y+(a)^{m}$, and each term of this sum is contained in $a I^{m-1}$ except the term $(P y)^{m}$. However we have $y^{m}+r_{1} a y^{m-1}+\cdots+r_{m} a^{m}=0$ from which we see that $(P y)^{m} \subseteq a I^{m-1}$. Thus $I^{m} \subseteq a I^{m-1}$. The other inclusion holds since $a \in I$.

Now consider $n \geqslant m$. By the first paragraph of this proof, we already have $P=\left(I^{m-1}: c\right)$ for some $c \in R$. Obviously $P=\left(I^{m-1} a^{n-m+1}: c a^{n-m+1}\right)$. However since $a I^{m-1}=I^{m}, a^{n-m+1} I^{m-1}=I^{n}$, and so $P \in A(n)$ for all $n \geqslant 1$.

Finally suppose that $R$ satisfies the altitude formula. Since height $P=\operatorname{dim} R>$ 1 , in order to show that $P \notin \hat{A}(n)$ for all $n>1$, in view of Corollary 4 , it is enough to show that $l(I)=1<$ height $P$. However the second paragraph of the proof shows that $(a)$ reduces $I$. Thus $l(I)=1$ as desired. 


\section{REFERENCES}

1. M. Brodmann, Asymptotic stability of Ass $\left(R / I^{n}\right)$, Proc. Amer. Math. Soc. 74 (1979), 16-18.

2. , The asymptotic nature of the analytic spread (preprint).

3. I. Kaplansky, Commutative rings, rev. ed., Univ. of Chicago Press, Chicago, Ill., 1974.

4. S. McAdam and E. Davis, Prime divisors and saturated chains, Indiana Univ. Math. J. 26 (1977), 653-662.

5. S. McAdam and P. Eakin, The asymptotic Ass, J. Algebra 61 (1979), 71-81.

6. M. Nagata, Local rings, Interscience, New York, 1962.

7. D. G. Northcott and D. Rees, Rectuctions of ideals in local rings, Proc. Cambridge Philos. Soc. 50 (1954), 145-158.

8. Cambridge Philos. Soc. 50 (1954), 353-359.

9. L. J. Ratliff, Jr., On prime divisors of $I^{n}$, n large, Michigan Math. J. 23 (1976), 337-352.

Departnient of Mathematics, University Of TeXas, Austin, Texas 78712 